\title{
汉语否定词形态句法类型的方言比较
}

\author{
刘 丹 青 \\ (中国社会科学院语言研究所)
}

提要 本文根据形态句法特征对汉语否定词进行一个跨方言比较, 主要考 察结果有: 1. 汉语古今南北普遍具有普通否定与有无否定 (存在否定) 的 词项差异, 但北中部两者声母不同, 普通否定为 [p-/f-], 有无否定为 [m-], 而华南两类同为 [m- ] 或其他斯辅音。2. 语法化使否定词泛化, 导 致词项减少; 词汇化使否定词与相邻的其他词发生熔合, 催生新的否定词 项。否定词频率高引起语音弱化, 为了强调否定义又常形成否定词的词形 强化。3. “否定词 + 谓语” 是汉语固定不变的语序。一些有疑问的现象并 不违背此语序。4. 西北方言的否定词强烈倾向于不管辖域大小直接用在谓 语中心上, 经常违背汉语普遍道循的语序一辖域对应律, 如 “甚不好” 表 示 “不很好”。这可以归因为附级化引起的核心吸附现象。5. 古汉语的 “不” (副词) “否” (叹词) 之别（对应于 not no）在普通话中都用 “不”, “不” 的副词和叹词两解正是历史上 “不” 重新分析为 no 义叹词的原因。 南方方言中普通否定词常没有否定叹词的功能。就吴语而言, 光杆动词、 形容词、副词普遍不具备普通话中的独用成句功能, “不” 类词的独用限 制只是这类现象的表现之一。

关键词 汉语方言 否定词 形态句法 语言类型 历史语言学

\section{Morpho-syntactic Typology of Chinese Negatives: A Cross-dialectal Survey}

\author{
Liu Danqing \\ Institute of Linguistics, China Academy of Social Sciences, Beijing
}

\begin{abstract}
This paper is a cross-dialectal survey of Chinese negatives, focusing on morpho-syntactic features. The main observations are as follows. 1. It has been a universal rule in Chinese to have different words for general negation and possession/existence negation respectively. In Northern (and Middle Chinese), the two kinds of negatives are different with [p-/f-] for general
\end{abstract}


negation and [m-] for possessive negation respectively whereas in the South both kinds are in the form of [m-]. 2. Grammaticalization makes negatives more general, hence lessening the size of the negative lexicon, while on the other hand, lexicalization has negatives fused with other words and brings about new negatives. The high text frequency of negatives weakens their phonetic forms, while stressing the negative meaning leads to reinforcement of negative forms. 3 . Negative-predicate ordering is the only order seen in Chinese, which is true over time and across dialects. Some seemingly complicated cases do not in fact violate this order. 4 . In Northwestern dialects, there is a strong tendency for the negative to be put immediately before the predicative verb/adjective despite its scope (e.g., 'very not good' means 'not very good'), which leads to a violation of the famous correlation between scope and order in Chinese. This should result from a head-attraction of clitics. 5. While Old Chinese employs bu and fou for the English adverbial not and the reply "no" respectively, the Mandarin negative $b u$ serves both functions. Sometimes, $b u$ can be analyzed either way. The double analyses could have lead to the reanalysis of $b u$ as not into $b u$ as no. However, in southern dialects like $\mathrm{Wu}$, there are no negatives for no. It might be because adverbial negatives in those dialects never serve as a short reply thus cannot be reanalyzed this way. This constraint reflects a more general rule in these dialects, i.e., a verb, adjective or adverb alone can hardly function as a short independent sentence.

Key words Chinese dialect negative morpho-syntax linguistic typology historical linguistics

\section{0. 小引：作为否定算子的否定词}

本文讨论的否定词 (negatives), 是指用来构成否定性命题的算子 (operator), 既包括单纯的否定算子 (如 “不”)，也包括语义上结合了其他成 分 (如 “别”、“更”、“覅”) 甚至动词本身 (如 “无” = “不 + 有”) 的否定 算子。类型学研究的 negatives, 还包括粘着的否定语素, 如日语的否定后缀 ない(a)nai (tobu 飞> tobanai 不飞) $)^{1)}$ 。

否定词的形态句法类型 (morpho-syntactic types) 是指否定词在形态（包 括构词) 和句法 (特别是语序) 方面的类型特征。本文通过跨方言的角度观 察汉语在否定词方面的一些共同类型特点和方言类型差异, 探讨其背后的人 类语言机制。 


\section{1. 形态类型: 否定词系统的词形分合和范畴整合}

从逻辑上说, 任何语言只需要一个否定词, 就能表达任何否定的意义。 事实上,一种语言 (方言) 中往往有不止一个否定词, 形成一个否定词系统。 否定词在词形方面的异同和语义分配方式，构成了语言的一种词汇 - 形态类 型特征。

\section{1. 普通否定与有无否定: 汉语的普遍特征及同中之异}

汉语从古到今在否定词项方面的一大特征是表示拥有兼存在的动词有专 用的否定词项，而不用普通的加 “不” 规则来类推。例如, “美、来、饮、在、 是、有” 的否定按规则应是 “不美、不来、不饮、不在、不是、不有”, 前 面各个组合都正确, 唯独 “不有”的意思不这样表达, 而要说 “无”（古） 或 “没 (有)” (今)。总体上这一特征自古而然, 至今犹然, 南北皆然。但 也发现了一些例外，特别是 “没” 并吞 “不” 的情况（详后）。

古代汉语和现代个别方言有 “不有” 或看似 “不有” 的说法。如《左传 - 昭公 16》有 “不有是事, 其能終乎? ”, 张敏 (2002) 指出这类都是假设句, 相当于北京话 “他不有个女儿吗”一类说法, 不是真正的 “不有”。此外, 我们在乌鲁木齐回民汉语方言语料中发现了“还有不有饭？”（刘俐李 1989:217)，这是用于正反问句的一部分，从书中看不出能否出现在其他场 合，但陈述句看来仍是使用 “没” 的: “有两个人穷的啊, 没吃的”（同上 $244)^{2)}$ 。

在 “不 无 / 没” 互补的共性下, 各方言也存在一大差异：普通否定与 有无否定是否同源。据此, 可以分出下面两大类型。

北中型 (包括官话、吴语、湘语、赣语等)：普通否定和有无否定的声 母对立, 分别为 $p$ (或吴语的 $f)^{3)}$ 和 $m$ (或其他鼻音变体)。这是由上古汉 语一脉相承的格局。

华南型 (闽、茎，客等): 普通否定和有无否定当为同源词, 都含有声 母 $\mathrm{m}$ (或其他鼻音变体), 如广州普通否定“唔” $\left[\mathrm{m}^{11}\right]$, 有无否定“布” $\left[\mathrm{mou}^{35}\right]$ 。 有无否定词可能多来自普通否定与 “有” 的合音, 有些现在还存在合音的迹 象 (参看张敏 2002 及所引文献, 覃远雄 2003)。对这一情况, 桥本 (1985 [1978]，3.2 节) 从语言地理学方面作了解释一一北方同阿尔泰的塞音类型, 华南同藏缅、壮侗的 $m$ 类型。而张敏 (2002) 则从动态 (历时) 类型学方面 提出了值得重视的新颖解释——存在否定词向普通否定词用法引申是人类语 
言常见而且可以循环发生的机制, 华南 $\mathbf{m}$ 型普通否定词是 $\mathrm{m}$ 型存在否定词 多次引申的产物。

北中型里的某些南方方言也以某种形式透露其普通否定词可能曾经是 $\mathbf{m}$ 型的, 至少有华南型的层次或底层。如温州虽然用 $\mathrm{f}$ 类普通否定词, 但在复 合词中有形容词 “毛” [mə] (坏, 不好), 是 “唔好” [m xə] 的合音 ${ }^{4)}$ 。宁 波话表示新使否定是 “唔毛 $\left[\mathrm{fm}^{22} \mathrm{mo}^{35}\right]$ ” (钱乃荣 1991:996) 或 “冒” [ $\mathrm{mo}^{53}$ / $\mathrm{mo}^{213}$ ] (李荣主编 2002 作 “莫”, 其实与 “莫” 的读音 $\left[\mathrm{mo}^{22}\right.$ ] 韵、调有异, 而且整个吴语区不见用 “莫” 表祈使否定的方言, 本文仍作 “冒”)。

\section{2. 否定词词形的类型差异}

1.2.1. 已然否定的词形类型。已然否定主要有这几种情况：用古代沿用的 “未” (张敏归入 $\mathrm{m}$ 类否定词)，见于华南; 用存在否定词 “没 / 无 / 右” 类 (官 话、彰语、湘语、中部南部吴语、华南地区多数方言), 用 $m$ 类普通否定 “唔” 加 “曾” 类构成的 “唔曾” 类及其合音（散见于个别繁方言、客家方言和闽 方言)，用 $\mathrm{p} / \mathrm{f}$ 类普通否定 “不 / 弗 / 勿” 等加 “曾” 类构成的 “不曾” 类及 其合音（主要见于北部吴语、微语, 后者见平田主编 1998:274), 但北部吴 语中的 “弗曾” 类开始被 “没” 类取代, 如在上海、无锡等地。

1.2.2. 根据预设而分出的主客观两类已然否定。一般是 “没 / 无” 类和 “未” 类并存的方言: 如广州粤语、福州闽语都有已然否定 “有 /无” (客观否定)、 “未”（主观否定）之别。广州话 “佢右去” 客观叙说没有发生“他去”这一 事件, 而 “佢未去” 则是说话人主观预期他要去, 而实际没有去。假如在事 件结束前这么说, 则可表示 “他尚未去” (但可能将要去)。华南以外方言多 无此分别, 有时用加副词 “还” 来表示 “尚未 V” 的主观否定 (他还没去)。 1.2.3. 可能式否定词的异同: 一般两者都用 “不”类否定词, 但福州话可 能式却用 “狯” (如 “讲狯完、买狯起”, 陈泽平 1998:177), “狯” 是 “未解” (不会) 的合音 (同上 175 页)。其他方言是用特定句法构式来表示可能情态, 而福州则在否定词中就包含情态成分 “解”。

1.2.4. 祈使式否定的词形类型: 多用普通否定加助动词表示, 如 “不要”、“不 用”、“唔使” (广州话)、“伓通 (福州话)”等。但由于合音而出现新的词形, 如 “别”、“覅”、“奋” 等。广州话的 “味” ( $\mathrm{mei}^{13}$, 别: 你咪去) 也像是合音, 但后字待考。

祈使否定词有时来源特殊或合音过程更加复杂, 导致一些更特殊的词 
形。

吴语中有些方言存在一些可能由 “休要” 合音或 “弗消” 脱落 “弗” 形 成的祈使否定词，如同里 (农村)、屯村等吴江北部方言的 “剿” [ zid $\left.^{24}\right]$, 七都等吴江南部方言的 “孝” [6 $\left.\mathrm{Gid}^{423}\right]$ (笔者调查)、双林话中的“啸” $\left[\mathrm{cis}^{53}\right.$ ]、黄岩话的“消” [6io $\left.{ }^{44}\right]$ (钱乃荣 1992:996)。“休” 是近代汉语中 由停止义动词虚化来的祈使否定词, “消” 是 “需要” 的合音。

云和吴语的 $\mathrm{yas}^{55} \sim \mathrm{ga}^{53}$ 是 “否定词 + 乐” [ $\left.\mathrm{fu}^{53} \mathrm{gaj}^{223}\right]$ (曹志耘等 2000:420 记作 “否要”) 的合音, 其中否定词声母韵母都不见于合音字, 只有声调有 所体现, 所以看不出否定词的痕迹, 成为新的否定语素 (“弗消” 变 “消” 也促成新的否定语素)。吴语中这个新否定词可以进一步失去声母读 “奥”, 如崇明方言 “奥去” (别去) (吴江话否定词 “奥” 只用于儿语)。

“莫” 从中古起有祈使否定的用法 (太田 2003:280), 北京话今不存, 南 方有不少方言以此为基本的祈使否定词, 主要集中在华中地区, 包括湘语(普 遍)、部分西南官话 (如武汉)、湘语周边的过渡方言 (如归西南官话的吉首 话, 李启群 2002:293; 归㖕语的安仁话, 陈满华 1995:161, 244)、黄孝片江 淮官话 (“鄂东方言”, 陈淑梅 2001:62, 但读阳去)。此外也见于梅县（林立 芳 1997:145-147)、连城 (项梦冰 1997:226) 等地的客家话及部分闽方言（如 福州话, 陈泽平 1998:179)。宁波吴语表示祈使否定的 “冒” $\left[\mathrm{mo}^{53} / \mathrm{mo}^{213}\right]$ 也 有著作写作 “莫” (李荣主编 2002 “莫” 条, 林、佐佐木、徐 2002:50), 但 是否为 “莫” 尚有疑问 (见上文)。用全量否定代词 “莫” 表示禁止与英语 全量否定词 no 加动名词（no +V+ing）如 no smoking（不准吸烟）表禁止可 能有共同的语义机制, 是一种回溯推理: 常规是假如不准吸烟, 那么就不会 有抽烟。回溯推理便是, 没有吸烟, 所以应当不准吸烟。于是用存在否定来 表示祈使否定。

\subsection{5. 其他词性的否定词}

“莫” 在发展出祈使用法的同时丢失了其否定代词的作用, 这是汉语在 否定词系统方面的一个显著演变。“莫”作为全量否定代词, 虽然不是直接 否定谓词, 但却具有构成否定性命题的算子功能。如 “(余掖杀国子, ) 莫余 敢止” (《左传》僖公 25 年), 即 “没有人敢于制止我”, 逻辑上等同于 “所 有人都不敢制止我”。而且从代词宾语 “余” 的前置语序看, “莫” 虽然是名 词性的, 但这种句子仍羊循否定句式的语序规则, 可见 “莫” 是否定词系统 
的一个成员, 意义上相当于英语中的 no 系代词 (nobody, none, nothing)。代 词性 “莫” 的消退非常彻底, 在各大现代方言中都了无踪迹, 也没见出现替 代词。各方言基本上都是用 “没有人”、“没什么东西” 这类动宾短语来表示 “莫” 的否定意义的。在这点上, 古汉语与英语属于同一类型, 而中古近代 以后的汉语及各方言属于另外一种类型了。

英语和古代汉语还有介词性的否定词, 即 without (I couldn't do it without his help) 和 “微”（微管仲, 吾其被发左社矣! - 《论语 - 宪问》), 现代类似的意思也要用动词来表示（假如没有管仲，……) 现代汉语各方言 也没见到这类否定介词，但现代汉语发展出了否定连词，如“以免”。

\section{3. 否定词词形分合的几点分析}

1.3.1. 从以上可知, 除了难以溯源的固有否定词, 否定词的形成机制主要 有语法化和词汇化两大类。

语法化就是从带有否定含义的谓词等较实在的词性发展为较虚的否定词 性。上文提到的近代汉语 “休” 的祈使否定用法是能找到确切的实词源头的 否定词。此外, 来自实词的否定词还有某些方言动词前 “懒”, 见于连城客 家话（项梦冰 1997:222），黄孝片江淮官话（陈淑梅 2001:62），来自 “獭得” 所含的否定义 "。“休”、“㭥”的否定词用法, 否定动词 “无/布”和 “没 (有)” 在不同方言中形成的副词用法, 这些都经历了语法化过程。语法化的发展趋 势是词项适用范围和搭配面不断扩大, 语义限制减弱, 因而否定词项会减 少。根据张敏所引的 Croft 的研究和张敏自己的研究 (2002), 最常见的语 法化发生在存在否定动词向普通否定词引申的过程中。张文所举的玉林方言 “布”就是一个突出的例子。玉林话“右”来自存在否定动词, 跟广州话的“布” 一样是 “无有” 的合音, 但经过语法化 “布” 已经变成一个全能的普通否定 词, 同时丢失了否定动词用法, 表示 “没有 NP” 要说 “右有 NP”。我们发 现还有比玉林 “布” 更加全能的方言否定词, 它既有玉林话 “布” 的各种用 法, 同时仍然保留了有无否定动词的用法, 如湖南嘉禾土话的 “没” [m $\left.\mathrm{m}^{22}\right]$

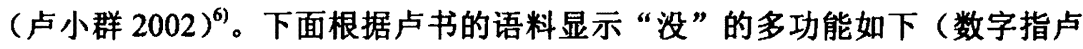
书页码):

存在否定动词: $\square\left[\mathrm{t}_{\mathrm{c}} \mathrm{ie}^{24}\right]$ 里还有饭没啦? ……酒过看者 [.tcie], 没咯 [.kə] 者。(151: 锅里还有饭没有? ……我去看了, 没有了) 到去三日没米煮。(158: 去了三天没米煮饭了) 
已然行为客观否定 (=奥语“布”): 打开眼睛又没蔍到 $\square\left[\mathrm{ia}^{51}\right]$ 什么。(171) 已然行为主观否定 (=般语 “未”)： $\square\left[\mathrm{lau}^{35}\right]$ 他还没食完吗？还没啦。 (150)

经历行为否定: 东边 $\square\left[\mathrm{pie}^{11}\right]$ 没 $\square\left[\mathrm{tai}^{11}\right]$ 过人。(153:东屋没有住过人) 现在状况否定: $\square\left[\mathbf{s}^{22}{ }^{22}\right]$ 记勒没记勒。(149: 你还记得不记得)

烟呀好，茶呀好，酒都没喜欢。(148: 烟也好, 酒也好, 我 都不喜欢）

系词否定：没是 $\square\left[\mathrm{kan}^{35}\right] \square\left[\mathrm{tcia}^{33}\right]$, 是 $\square\left[\mathrm{kag}^{51}\right] \square\left[\mathrm{tcia}^{33}\right]$ 。(126： 不是这里, 是那里)

能愿助动词否定：酒应该没应该来？(144: 我应该不应该来？) 形容词否定: 来听一下 $\square\left[\mathrm{ke}^{33}\right]$ 朵花香没香。(128: 来闻一下这朵花香 不香)

差比句否定：酒没咯 [.kə] $\square\left[\operatorname{san}^{22}\right]$ 壮。(142: 我没有你胖)

祈使否定：你就没要告诉隔 $\square\left[\mathbf{s s n}^{33}\right]$ 人者 [.tcie]。(169: 你就不要告 诉其他人了)

可能补语否定: $\square\left[\mathrm{ka \eta}^{35}\right]$ 是生各 [.kə], 食没勒。(154:那是生的, 吃 不得）

否定词的词汇化主要是指否定词与其他语素的熔合 (fusion) 造成新的否 定词项, 这些经过揢合的词就成为新的否定词形或否定语素。词汇化会导致 否定词词项繁复，如 “没、不、别、霄”, 福州话 “唔、伓、硷、未、莫”, 苏州话 “唔拔、勿、璔、顷”, 广州 “唔、布、未、咪” 等。一种特殊的词 汇化是 “否定词 + 要 / 消” 组合脱落否定词, 剩下 “要 / 消” 成为新的否定 词（见 1.2.4）。

语法化和词汇化是影响否定词系统的两大相竞 (competing) 机制。从语 义上看, 语法化是泛化 (generalization), 词汇化是专化 (specialization)。 两者背后各有功能动因。语法化的动因是类推和聚合经济性一一使用尽可能 少的否定词, 在不同的否定结构中使用同样的否定词, 以便减少习得和记忆 规则的负担。词汇化的动因是高频简化和组合经济性一一高频词信息量会降 低, 高频组合中两个要素的紧密度和预期性都会增强, 信息量相应降低, 这 些都会促使有关组合发生熔合, 减少发音的用力和时长, 符合组合的经济性。

语言、方言总是处在这两种相竟力量的某种中间位置, 否定词的词形数 
量不会无限膨胀, 也不一定能长期维持一个否定词单骑走天下的局面。在历 时演变中会维持一种归一与歧异的动态平衡。例如上举北京话、福州话、苏 州话、广州话的否定词系统, 总数都是 $4 、 5$ 个。

1.3.2. 否定词与其他词的合音导致新的否定词中含有其他的范畴义, 如情 态、言语行为功能 (语气)、时、体等, 使否定词不是挂单的词项, 而形成 一个系统。这种情况在语言中是很常见的。标准英语中谓语的否定词似乎只 有一个 not, 但熔合孕育新否定词形的过程已露端倪, 如 don't, can't 等形式 中已经难以自然地分离出一个否定词或否定语素 (can't 的 $\mathrm{t}$ 基本不发音, 而 前面的 a 的音色与肯定式 can 中的 a 有异, 据史皓元先生 R. Simmons 恵告, can 和 can't 基本上只靠元音分辨)。而在英语某些方言中, 深度的揢合（整 个否定词音段成分消失、只带来前面音节的改变）已经导致整整一批否定词

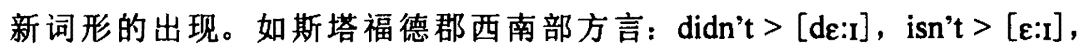
doesn't/don't $>$ [dou], wasn't > [wo:], shall not $>$ [ $\mathrm{ja:l}$ ], aren't $>$ 变 [æ:], will not> [wou] (参看 Kortmann1999, 刘海燕译 2004)。

1.3.3. 否定词系统还存在另一对矛盾因素的互动和平衡, 即弱化与强化。 否定词因其出现频率高而容易弱化, 于是出现合音等熔合现象, 造成音节缩 减, 如上所举。另一方面, 否定词因其提供的信息重要, 而时常被加上其他 语素进行强化, 造成音节增加。法语 pas 就是一个增强否定功能的状语, 最 后因语义感染自身变成了否定词。古代汉语 “无” 即是 “有” 的否定, 但又 产生出 “无有、未有” 等双音形式, 其中 “无有” 没有增加任何新的语义, 就是一次强化。从读音上看, 写作 “没” 的汉语否定词在很多方言中很可能 是 “无有” 或 “无得” 的合音（详见覃远雄 2003), 合音又是一种弱化。假 如再说成 “没有” 或 “没得”, 则又是一次强化, 仔细分析其内部要素是 “无 有有 / 无有得 / 无得得 / 无得有”之类。又如 “未” 已是对已然性的否定, 但古代汉语中又有“未尝、未曾” 等复合强化形式, 客客方言也有“未曾” 的说法。再如 “休” 本是 “不要”之义, “休 V” 足以表示祈使否定, 但后代 又有说 “休要” 的, 太田 (2003:280) 认为这是由 “不要” 类推出的 “误用”。 不过, 强化叠加和强化弱化交替在语法化过程中是十分常见的（参阅拙文 2001 ), “休要” 这类叠床架屋并不奇特, 说不上是 “误用”。强化和弱化的 反复交替可能是方言否定词词形歧异的重要原因之一。

否定词在强化弱化过程中还常伴随语流音变, 这会导致词形的进一步复 
杂、加剧方言间甚至相邻方言间在否定词形上的分歧。如江浙沪交界处的北 部吴语中有无否定词就有 “呒拨” [m po? （无锡、苏州，也作 “呒不”)、“嗯 得” [n tor]（昆山）、“呒没” [m mor] (上海、嘉兴) 等形式, 从某些写法看, 似乎还是两个否定词的连用，其实来源都是 “无得”，读音和写法的歧异是 语流音变的产物。苏州无锡的 “呒” 是反映微母字 “无” 早期悬声母的白读 （文读为 [vu] ), 在昆山受后字 “得” 的逆同化而变成同部位宜音 “恩” [n]。 在苏州上海, 则 “得” 字的读音由于受 $\mathrm{m}$ 的不同程度的顺同化而形成双唇 声母的 mə? 或 pə? 。苏州的 “呒拔”、上海 “呒没” 在早期文献中都有作 “无 得” 的 " , “无得” 就是语流音变前的词形。“呒不、呒没” 等写法容易让人 认为其中含有否定语素 “不 / 没”, 其实都是 “得” 的音变形式 (邻近的江 淮官话也普遍用 “没得”一实际可能是 “无得得”)。绍兴话 “嗯有” [n jour] 则是用 “有” 强化 “无” [m] , “无” 的双唇鼻音再受 “有” 的舌面 半元音 [j] 的同化而趋近成为舌尖鼻音 “嗯”, 就是古代汉语的 “无有”。

\section{2. 句法类型: 否定词的语序和辖域问题}

\section{1. 否定词的前置 (后向否定) 与后置（前向否定）}

否定词本身的语序问题, 就是否定词作为否定算子（negative operator） 与其辖域 (scope) 的位置关系。

虽然否定词在语义上是作用于表达命题的整个小句的, 但在句法上却通 常只是作用于谓语部分即 VP。Givón (1984:325-326) 以话语分析证明有定主 语绝大部分不在否定词的辖域内。类型学调查也显示否定词与 VP 而不是与 包含主语的小句整体关系更紧密。根据 Dryer (1988) 对否定词语序的语种库 统计, SVO 语言中否定词 (Neg) 的语序分布依次为:

SNegVO: 47 种语言, 13 个语系（其中 12 个语系中该句式占多数）

SVONeg: 13 种语言, 4 个语系 (其中 1 个语系该句式占多数)

NegSVO: 4 种语言, 3 个语系

SVNegO: 3 种语言, 1 个语系

显然, 占绝对优势的是第一种语序类型, 否定词以 VP 而非整个小句为辖域, $S$ 通常不在其辖域之内。再看 SOV 语言:

SOVNeg: 64 种语言, 18 个语系

SONegV: 39 种语言, 15 个语系 
NegSOV: 8 种语言, 5 个语系

SNegOV: 6 种语言, 3 个语系

在占优势的第一种语系类型中, 否定词辖 VP 和辖整个小句的语序是一致 的, 位居第一是两种力量的合力。位居第二的语序, 否定词显然只能辖动词 不能辖主语。而只能理解为辖整个小句的 NegSOV 位居第三, 数量上明显 处于少势。这也显示否定词的常规是以不包括主语的 VP 为辖域。

再从 VO 内部看, 否定词的绝对优势是直接与动词组合, 在 SVO 语言 中占绝对优势的第一种类型和在 SOV 语言中占绝对优势的第一、二种类型, 否定词都直接跟动词组合。动词（尤其是带有定式谓语屈折成分的动词）是 小句结构的核心, 更是谓语 VP 的核心, 否定词加在谓语的核心上, 以否定 核心来否定全句是非常合理的, 是语言的一种嵀贼满王的策略。此外, 否定 词通常较短小, 独立性相对较弱, 最好有一个依附对象, 自己充当其附缀 (clitic), 而作为命题算子的否定词依附在命题的核心动词上当然比依附在动 词所投射的论元上或修饰成分上更加合理。否定词强烈的附缀化倾向甚至可 以用违背否定词辖域作代价 (见下)。

由此可见, 在语序类型学中, 考察否定词的语序, 主要是考察否定词相 对于谓语核心的位置。根据这一参项, 否定词的位置有前置和后置两种。

下面的俄语句子体现了否定词前置（即后向否定）的情况:

Он не знает русского языка.

他不懂俄语他不情俄语”

下面的德语句子体现了否定词后置（即前向否定）的情况：

Er făhrt nicht Auto.

他驾驶不 汽车 “他不驾驶汽车”

在不少语言中, 前置和后置两种语序并存, 可能各有其适用范围。在现 代英语中, 谓语的否定词不能加在实义动词上, 而必须加在原有或添加的助 动词上, 相对于助动词, 这些否定词都是后置的, 如 She cannot work（她不 能工作), not 加在 can (原有) 之后, 前向否定, John doesn't love her (约 翰不爱她), n't 加在 does（添加）之后, 前向否定。从实义动词角度看, 否 定词似乎也是后向否定, 但带助动词的句子中时和一致关系等屈折成分都加 在助动词上, 是谓语的核心, 实义动词只是不定式。所以, 这些否定词都应 分析为后置的。不过, 在非主句谓语的层面, 否定词又是可以后向否定即前 
置的。如 I prefer not to go ( 我倾向于不去), not 后向否定 to go, 是前置型的。 两种否定方向的并存突出表现在助动词上。据 Palmer (1974:137), may not V, will not $\mathrm{V}$, mustn't $\mathrm{V}$ 中的否定要素都是后向否定实义动词 $\mathrm{V}$ 的, 而 can't, needn't 中的否定成分都是前向否定 can 或 need 的。

从已有材料看看, 汉语从古到今、从南到北, 不管在什么层次上, 否定 词向来是前置性的, 即只用来后向否定。如:

先秦汉语: 人不堪其忧，回也不改其乐。(《论语·䔨也》)

广州藇语: 佢唔中意食生果。(他不喜欢吃水果)

宁波吴语：尔酒目吃。(你别喝酒)

这些情况体现了汉语语序固定且稳定的一面。

有两种情况的否定方向看起来不那么直接, 需要提出来讨论一下。

\section{2. “ $\mathbf{V}$ 得/不 $\mathbf{R}$ ” 可能式中的否定方向问题}

普通话表示可能义常用 “ $V$ 得 / 不 $R$ ” 可能式 ( $R$ 代表结果成分), 而且 这对格式中否定式远比肯定式常用（刘月华 1980）。从 “看清～看得清～看 不清” 的格式对应看, “看得清” “看不清” 是动结式 “看清” 的可能式, 由 VR 式 “看清” 中间插入 “得 / 不” 构成, “得” 和 “不” 形成肯定否定相对 的标记，相对于 “看清” 来说否定词 “不” 似乎是中加成分，其作用域似乎 非前非后，而是其两边的成分。事实上“得” 和 “不” 句法上并不是对称的。 “得” 是加在 $V$ 上的, 其层次是 “ $V$ 得 $/ R$ ”。“ $V$ 得” 在汉语中本来就能单独 构成可能式, 如 “和尚动得, 我动不得? ” (鲁迅《阿 $Q$ 正传》), 而 “得 $R$ ” 则不成立, 不能说 “得清”。“不” 是加在 $R$ 上的, “看不清” 的层次是 “V/ 不 R”, “不 R” 是合格的语法组合, 如 “不清”。联系汉语史来看这一点更加 明显。可能式的肯定式和否定式非出一源, 前者比后者形成时间要晚, 句法 表现也不一样（参阅太田 2003[1958］，16.6.3 节), 突出表现在其当初带宾 语的不同格式上:

肯定式

$V$ 得 OR (看得他清)

*VO 得 $\mathrm{R}$ (*看他得清)
否定式

*V 不 OR” (*看不他清)

VO 不 R (看他不清)。

以上结构差异清楚地显示 “得” 的后置性和 “不” 的前置性。现在普通话的 “V 得 $R O$ ” 和 “ $V$ 不 RO” 的表面对称是两种可能式因为意义对应而发生格式 类化的结果。此外, 南方方言中倒确有 “VO 得 R/V 不 OR”。但吴福祥 
(2002) 通过比较研究指出, 有 “VO 得 $R / V$ 不 $O R$ ” 的方言必有 “ $V$ 得 $O R /$ $V O$ 得不 $R$ ”, 而有 “ $V$ 得 $O R / V O$ 得不 $R$ ” 的方言则不一定有 “ $V O$ 得 $R / V$ 不 $O R$ ”。“VO得 $R / V$ 不 $O R$ ” 配对是一种晚于 “ $V$ 得 $O R / V O$ 不 $R$ ” 配对的层次, 是方言类推将两种格式的表层差异拉平的产物。因此, 在 “ $\mathrm{V}$ 不 $\mathrm{R}$ ” 式中, 否定 “不” 仍是前置性的, 与其在汉语中的一贯表现完全一致。

上面的分析, 特别是 “得” 和 “不” 在可能式中的不对称, 在某些方言 事实中得到更清楚的显示。在山东、河南的一些官话和晋语方言中, 可能式 的肯定式和否定式本身在不带宾语时就是不对称的, 如下:

肯定式

否定式

VR 了

V 不 $R$

(聊城: 考上喽 | 德州: 干完溜)

（聊城: 考不上 | 德州: 干不完） 上面肯定可能式中的 “了” 有各种读音变体, 故又写作 “喽、劳、溜、哩” 等, 但这些读音都等同于各自方言中体标记 “了，”的读音（据钱曾怡主编 2001: 284-285)。在这些方言中, 我们看到的是 “VR 了”与 “V 不 $R$ ” 的极性对立, 去掉核心动词就是 “ $R$ 了”与 “不 $R$ ” 的对立, “不” 是加在 $R$ 上的否定词, 与结构助词 “得” 无关。“不” 的前置性更加显著。

\section{3. 是非疑问句末否定词的否定方向问题}

“不” 在一些方言中有一种是非疑问标记即疑问语气词的用法, 相当于 普通话的 “吗” ——实际上就是重走从否定词 “无” 到疑问语气词 “吗” 的 重新分析之路。山东方言中这样的现象较常见, 如（引自钱曾怡 2001: 298):

聊城：你愿意去不? | 你想开喽不?

东平：吃饭啵？|脸红皮？(“皮”系这种“不”的一种写法)

㫡庄: 学习不? | 吃饭不?

与本文有关的问题是, 这里的 “不” 是前置的算子还是后置的算子。

从语言类型学的角度看, 正反问句并不是一种独立的问句类型, 在共时 平面, 它是用选择问句的形式起是非问句的功能, 在历时平面, 它是选择问 句向是非问句转化的过渡环节, “不” 在这个过程中由否定算子向疑问算子 转化。有些方言用 “皮”一类同音字来记录这种 “不”, 说明在该方言的语 言心理中它已重新分析为疑问语气词了。另一些方言则还处在更接近否定词 的阶段, 作为 “不 VP” 的省略形式, 所以在 VP 和 “不 VP” 之间还要加连接 
性虚词, 如寿光 “这个人是老师呕不”、淄川 “看电影响不” (加连接虚词的 正反问句在西北方言也多见)。在转化之前, “不” 作为否定算子是前置性的, “不” 是 “不 VP” 的省略形式: 在转化之后, “不” 作为疑问算子是后置性的, 是作用于前面的肯定命题句的。在转化过程中, 存在着双重分析的阶段。即 使在这个阶段, 它作为否定算子仍是前置的。因此, 疑问算子一律前置的汉 语通则在这种情况下依然有效。

\section{4. 否定词在多项状语中的语序和辖域}

除了否定词和谓语核心的语序关系外, 汉语否定词还有一个至今还较为 忽略的语序问题: 否定词在多项状语中的位置问题。

汉语否定词和程度副词自古以来一直莩守语序和辖域一致的通则——离 核心越远的修饰或限制成分其辖域越大。如 “很不高兴”, “很” 在前取广域, 修饰整个 “不高兴”, 而否定副词 “不” 在后取窄域, 只修饰 “高兴”。假如 要让否定词 “不” 取广域, 必须让 “不” 位于程度副词之前, 说成 “他不很 高兴”。汉语的各大方言基本上都道守这一通则。但是我们发现, 在这一点 上也出现了一些显著的例外。

王森（2001）描写了西北陕甘回民的后代一一中亚东干人所说的东干话 不同于普通话的诸多语序特点, 其中不少就与否定词有关。据初步了解, 这 些语序特点也基本存在于关中、甘肃等东干族出发地的中原、兰银官话方言 中。由唐正大新近的调查 (个人交流) 证实, 关中方言中存在这类语序特点 （下文 “<关中>” 即唐调查的关中方言的同类例句）

根据王文介绍, 东干话否定副词 “不、没、䨘” 除了出现在能愿动词前 及在个别情况下出现在介词短语前之外, 一般总是紧靠谓语中心语前面, 其 他词语充当的状语都在否定词前, 跟普通话相反。他将此类情况总结为 5 种, 这里略作转述:

A. 表示轻度否定时, 用 “程度副词 + 否定副词” 的语序, 意义上相当 于普通话 “否定副词 + 程度副词”, 如 “甚不高” 表示不太高, “很很 地不热” 表示 “不太热”。<关中>：“这个电影甚不好”（不很好）、“张 三今儿胃口不好, 甚没吃” (没吃多少)。

B. 范围副词 “光 / 都 + 否定词” 表达相当于普通话 “否定词 + 光 / 都” 的意思, 如 “光要燋哩” (不要只是看)、“都也不是好的” (也不都是好 的)。 
C. 表示处所、方向、对象、协同、方式等多种介词短语都要放在否定 副词前。如 “把肉没打上 “(没买到肉)、“在园子呢不睡” (不在园子里 睡)、“她给男人没给钱” (她没给她丈夫钱)。<关中>: “我到西安没去”、 “张三把猪没杀”、“张三给他妈不吃好的”。

D. 副词 “白、可再, 又、胡”、形容词 “多”、代词 “这么家、那么家” 都要放在否定词前。如 “白不养一个穷汉” (不白养一个穷人)、“他那 么家说呢, 可那么家不干” (他那样说, 却不那样干)。《关中> : “我再 不吃咧” (我不再吃了)。

E. 依此递加的多个状语也要放在否定词前, 如 “把儿子再要吓下哩” (别 再把儿子也吓着了)、“为啥把这个事情给我早些儿不说勒” (为什么不 早点儿给我把这个事情提出来呀! )。<关中>: “张三叫李四也没占下啥 便宜” (张三也没让李四占什么便宜)。

在以上情况中, 否定词基本上都处于广域 (wide scope), 但却出现在受 它支配的句法成分的后面。特别是辖域明显小于否定词的那些成分, 如 A 类中的程度副词、B 类中的全量限定副词、C 类中的 “把” 引导的受事论元、 “给”引导的与事 (接受者) 论元等, 在普通话和其他方言中都是严格按辖 域一语序对应的通则出现在否定词之后的。东干话及关中方言等的这些特殊 语序, 直接有悖于汉语语序与辖域完美对应的通则, 需要作出解释。

刘俐李（1989:214-215）曾简短提到过乌鲁木齐回民汉语方言中的类似 现象:

老师的话他好好儿不听 | 把功课不做完 | 给你没买 | 我的Y头也给你不 给, 也给你不给官做 刘俐李推测, 这些语序现象可能都是受了维吾尔语的影响, 因为维语都用动 词上加否定式词尾来构成否定式。如:

$\begin{array}{lcc}\text { u } & \text { yahxi } & \text { ixliməydu } \\ \text { 他 } & \text { 好好 } & \text { 干-否定 - 第 III 人称 }\end{array}$

在否定词直接加在动词上的情况下, “好好”一类状语只能是位于否定成分 之前了。

刘俐李的推测可能有些道理, 但也遇到一些困难。

从外部条件看, 类似的语序现象在西北地区陕甘宁疆的汉语方言中广泛 存在, 其中的关中方言等末必有直接与维吾尔语大量接触的机会, 不像是维 
吾尔语的影响。

从语言内部结构看, 此说也有几个困难。其一, 维吾尔语否定后缀在动 词后, 前面的其他状语还是能直接与动词相接的, 结构与西北诸方言中的否 定词特殊语序并不相同, 难以看出直接影响。其二, 东干话和它的源头方言 关中方言等的特殊语序并不限于否定结构。有些特殊语序和否定词的特殊语 序应当是同类的，但并不涉及否定式。如，肯定句多个状语的语序倾向是， “单音节词紧靠在谓语中心语前; 介词短语或其他多音节词语在单音节词前; 介词短语在其他多音节词语前面; 几个单音节词连用时, 形容词在副词或能 愿动词前” (王森 2001)。王文例子甚丰, 这里略引数例, 括号后是引者补 出的普通话的相关语序:

给他要找奶子呢（要给他找奶呢）

他拿括头也打呢, 拿脚也踢呢。(他又拿拳头打, 又拿脚踢)

车太难走, 我们打车上就跳下来, (……我们就从车上跳下来, )

我把哈人一定拘死。(我一定把哈人勒死)

我们还多要盖工厂呢。(我们还要多盖工厂呢)

娃们乱都喊的呢。(孩子们都乱喊着呢)

否定词的特殊语序, 也被王森归为上述 “总的倾向” 下的具体表现之一。

从王森对特殊语序现象的概括可以看出, 这些特殊语序一是与音节数有 关一一单音节或相对更短的形式紧靠动词, 二是与词类属性有关一一副词或 能愿动词紧靠动词。这些倾向于紧靠动词的状语 (上述东干话例中黑体者) 在维吾尔语或其他阿尔泰语言中并非都用动词的形态来表示, 例如表示全量 的 “都”、表示类似的 “也” 都难以在阿尔泰语言中找到相应的动词形态。

我们发现, 上面所说的包括否定式在内的种种语序现象, 都可以用一条 “核心吸附” 的总规则来解释。就是说, 在东千话及汉语西北诸方言中, 谓 语的核心会将状语或情态 (modality) 成分中独立性弱的成分吸附过来, 紧 挨在谓语核心之前。所谓独立性弱, 就是短小轻弱。所以, 核心主要吸附的 对象是单音节成分。在没有单音节成分的情况下, 双音节成分也可能被吸 附, 如上面例中的副词 “一定”。介词短语是这些状语中最长最复杂的, 因 而也就不会发生吸附现象。当存在几个单音节成分时, 虚化程度高、读音更 弱的成分优先被吸附。形容词意义比能愿词 (属情态成分) 和副词实在, 读 音也会比能愿词和副词强, 因此同现时能愿词和副词优先被吸附。否定词由 
于出现频率高, 在语流中常常弱读, 因而是最容易被吸附的对象。

正是西北方言的这条核心吸附规则, 打乱了汉语辖域与语序高度一致的 常规, 造成了语序与辖域的错位。不过, 这种核心吸附现象, 也不完全是这 些方言的独特规则。有一系列的常见机制可以作为这一核心吸附的支撑。最 明显的是附缨化 (cliticization), 就是一些词 (多为功能词) 部分失去其独 立性成为依附在实词上的附缀 (clitic)。就悉的例子如英语 I'm 中的 $\mathrm{m}$ (<am), He's finished it 中的 s (<has) 等, 都属于附諘。附缀化的对象都是 出现频率高、意义淡化、语音弱化的单位。附缀化有些不发生语序的变化, 像上面的英语例子: 有些则同时伴随语序变化, 改变与核心的位置关系和距 离关系。在语法化理论中, 附缀化常常是独立的词重新分析为词缀的第一 步, 形态复杂语言的很多词缀可能就是由最初的附缀一步步发展来的, Trask (1996/2000:118) 从法语口语正在发生的伴随语序变化的附缀化等现象 推测, 巴斯克语、蒙古语中的动词形态很复杂, 可能就是由独立的词经过附 缀阶段而发展来的。比较法语中的下面三个句子 (见 Trask 上引书页), 其 中第 3 句是当代口语句子:

Jean donnera le livre à Marie.

人名将给（定冠）书给人名 'Jean 将送这本书给 Marie' Il te le donnera.

他 (主) 你(宾) 它 (宾) 将给 “他将把它送给你”

Jean, il te le donnera, le livre.

人名 他 (主) 你 (宾) 它 (宾) 将给 (定冠) 书 'Jean, 将把它送给你, 这本书” 在第 1 句中, 主宾语都是实义名词, 分别在给予动词之前和之后, 体现 SVO 语序。在第 2 句中, 主语、直接宾语和间接宾语都是非重读人称代词(法 语另有重读代词在语用需要时用), 在法语中都表现为附叒, 而附缀性代词 都要用在动词前, 语序变成了 SOV。在体现当代口语的第 3 句中, 主语和直 接宾语本有实义名词出现, 但口语中还是使用了与实义名词同指的非重读代 词, 这种附缀代词已没有独立论元的句法地位, 语序也不同于实义名词, 它 们正在成为加在动词上的与主宾语保持一致关系的词缀。

东干话 “特殊语序” 中后置的否定词或其他状语和情态成分在几个方面 都符合上面这些附缀化的特点: 出现频率高, 语音简短而弱化, 意义淡化, 紧贴实词, 改变语序以紧靠核心实词。因此, 我们相信东干话及相近方言中 
的状语类的特殊语序是一种类似于附缀化的现象, 可称为准附缀化。附缀化 本身是人类语言中常见的与共时历时都相关的句法现象, 在汉语中也不少 见。只是其他方言中的附缀化或准附缀化仍受辖域规则（包括否定词辖域） 的制约, 其优先地位在辖域规则之下, 而东干话的准附缀化已可以突破辖域 规则的约束, 从而打破了辖域与语序的一致关系。

回过来看, 刘例李的前述推测也非全无道理。自古以来西北地区是阿尔 泰语言与汉语频繁密切接触的地区。阿尔泰语言也确实将很多由汉语副词、 助动词表达的内容用动词上的形态成分来表达, 包括否定、属于 “式” 范畴 的可能和愿望、属于时范畴的过去现在将来, 等等, 参看力提甫主编 (2002:45-46)。特别值得注意的是, 力提甫书指出, 阿尔泰语系突厥语族的 一个发展趋势是 “表示动词的各种语法意义的语法形式不断增多, 越来越丰 富”, 而从历时角度看, 这些形式在成为动词的形态之前首先会被动词吸附 成为紧靠动词的附殕形式。考虑到这些情况, 再对照汉语, 它从古到今、从 南到北一直严守辖域与语序一致的规则, 唯独在西北地区出现很多因核心吸 附而突破这一规则的语序现象。因此, 不排除东干话等西北方言的特殊语序 与阿尔泰语言的可能联系。只是这种可能的联系还需要通过细致扎实的语言 比较和历时分析才能真正确定。

\section{3. 句法类型: 否定句的独立成句问题}

否定词独立成句的能力在汉语否定词类型研究中尚未引起足够重视, 近 来有些著述曾有所关注。詹伯慧主编 (2002:81) 在介绍广州话 “唔” 时说 “相 当于 “不”, 但是 “唔”一般不能单用”。拙文 (2002) 注意到吴语缺少相当 于 “不” 的单说型普通否定词, 上海话新派的 “晤没” 则开始有了独立成句 的新用法。这里将结合更广阔的类型背景来探讨这一参项上的南北差异。

这个问题要从单说的 “不” 和否定副词 “不” 的关系说起。看下面的例 子:

甲: 你去吗？／你去不去。

乙: 不, 我不去。

对于答句中的第一个 “不”, 可以有两种分析法: 一, 看作 “我不去” 的省 略形式, “不” 仍是否定副词 (古汉语 “不”, 英语 not), 二: “不” 是独立 的否定叹词 (英语 no）或否定代句词（古汉语主要用 “否”), 是对整个命题 
的否定。在上例中, 两种分析都成立; 在另一些情况下, 只能分析为独立的 否定叹词或代句词, 如 “小张也去了。一不, 他没去”。这里的 “不” 无 法补充成 “他不去”。

现代汉语的两个 “不” 同形, 是本来只作否定副词的 “不” 寔食了 “否” 的领地, 发展出代句词的用法。这种取代的前提是否定副词 “不” 可以省略 谓词单用, 产生上述两可分析中的第一种分析, 然后经过上例这样的双重分 析后再重新分析为代句词。而一些南方方言没有相当于“否”或 no 的否定词, 首先是因为普通否定副词 “勿”、“唔” 等不能脱离所否定的谓词单用, 因而 也无法由此发展出否定代句词。而普通否定副词不能单用的现象至少在吴语 中有着更加广泛的类型学基础。

以苏州话和老派上海话为代表的北部吴语没有能单独成句的普通否定 词。如下面的北京话和苏州话的比较:
京: - 你抽烟吗?
- 不。
苏: 一 倷阿吃香烟?
- 小张到广州去了。
- 勿吃。(*勿。)
- 不, 他到深圳去了。
- 小张到广州去哉。
- 勿是, 俚到深圳去哉。(*勿, 俚到深 圳去哉。)

由上例看到, 对于否定性的应答, 苏州话不能使用单独的普通否定词。有两 种策略可以使用。假如有合适的谓词, 否定词会和谓词一起出现, 如“勿吃”。 假如没有合适的谓词, 则起用判断动词, 如 “勿是”。“覅、晹” 等熔合了其 他语素的合音字和“呒拨” (没有) 等存在否定动词及更加复杂的否定习语 (如 “来勿及”）则可以独立成句。

在苏州话中, 不能单独成句的情况远非否定词所独有。其他一些在北京 话中可以单独成句的副词、能愿助动词和谓词, 在苏州话中也不能单独成句 或回答问题。包括系词 “是”, 其否定形式可以单独成句, 如上举 “勿是”, 但肯定形式就不能单独成句, 必须加上本义为 “的” 的语气词 “个”。其他 不能单独成句的词往往也要靠 “个” 或其他语气词如 “啘” [uə] 来成句 (下 面用括号上加星号表示删除 “个” 就不合格), 如:
京: 一 他儿子聪明吗?
苏: 一 俚哚儿子阿聪明。
- 聪明。
- 聪明*(个)。
- 他会不会说英语?
- 俚阿会得讲英语? 
一会。

- 我可以见见他吗?

- 可以。

- 他今天回家吗?

- 可能 / 也许。

- 你介绍我认识他吧!

- 好。

- 他是不是大学生?

一 是。
一 会得 *(个)。

- 我阿可以见见俚?

- 可以*(个)

- 俚今朝阿转?

- 作兴 *(个)。

一倷介绍我认得俚吧！

- 好*(个)。

一俚阿是大学生?

- 是*(个)。

反过来, 假如以上北京话独词答语都带上 “的”, 多半也会不太自然。这一 对比说明, 吴语的谓词和情态性副词是语义上更加赤裸 (naked) 的词项, 需 要加上成句所需的语气、情态成分如 “个” 来帮助成句 ${ }^{8)}$ 。也因为这种类型 上的需求, 吴语的 “个” 语法化为比 “的” 更虚、更具有句法强制性的句子 完形标记。有趣的是, 一些有情态语气作用的成分可以帮助其他词语成句, 自己却不能成句, 还需要其他情态语气成分帮助。如 “勿” 和 “是”, 两者 可以帮助对方成句, 也可以帮助其他词语成句, 上述苏州话答句假如用 “勿” 否定, 都可以不需要 “个” 而成句, 如 “勿聪明、勿好” 等。但是 “勿” 和 “是” 自身却不能成句, 必须说成 “勿是、勿啘 [uə]、是个、是啘” 等才能 成句。

北部吴语普通否定副词没有独立成句能力, 也就没有了重新分析为否定 代句词的句法环境, 这是由吴语谓词副词整体上成句功能弱的类型特点造成 的。与此相关, 当代新上海话正在形成用有无否定动词 “呒没” 充当命题否 定代句词的局面, 代替原来的 “勿是” 等短语（详刘丹青 2002, 4.2)。这一 新发展在功能上也许受到普通话否定代句词 “不” 的影响, 但词项上却选择 了相当于 “没有” 的存在否定词 “呒没” 而不是更加对应的 “勿”, 这是因 为 “呒没” 动词性强、在上海话中本来可以独立成句, 现在只是从存在否定 引申到其他的命题否定。此外, 这一变化也符合人类语言的常见机制。英语 用语命题否定的 no 虽然不是动词, 但在语义上仍具备存在否定词的作用, 如 I have no choice, nobody, nothing, 这些词句中的 no 在上海话中都要用 “呒 没” 来翻译。闽南话也用 “无” 构成独词否定句, 如李如龙 (2001) 所举 “汝 
明旦若卜出差? 一无, 我唔去” (你明天出差? 一不, 我不去了)。由存在 否定词发展成否定叹词/否定代句词, 这是上海话、闽南话和英语共有的语 法化道路。

\section{<注〉}

1) negatives 译为否定算子相当精确，可以包括否定词和否定语素，只是技术味太重些。

2）有同行告知云南典州有些汉语方言（包括少数民族说的汉语）有 “不有” 的说法, 但 尚未找到确切材料。和乌䡒木齐一样, 这些也是少数民族语言通行地区, 不排除是语言 频慗接触的结果。

3）根据潘悟云 (2002), 吴语写做 “弗、勿” 等的 f-/v- 类否定词, 其实都是 “不”, 来 自 “不”的 《广的》“方久切” 及其后来的促化形式。所以我们将吴语也归入 “北中型”。

4）温州话该义的 “毛” 条收入李荣主编《现代汉语方言大词典》(2002) 及其温州方言分 卷本 (游汝杰、杨乾明编), 其注释是: “(1)缺点多的; 使人不满意的 (与 “好” 相对) ……(2)品质恶劣的; 起虍为作用的……(3)使变坏……”. 未提合音问题。合音的解释是 潘悟云先生提供的。

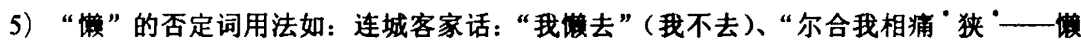

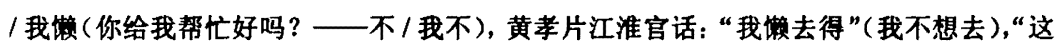
个话我濑对你说得” (这个话我不想对你说)。意义上还多带主观意愿义，未彻底虚化到 “不” 的程度。广州话 “阔佬馍理” 中的“塻” 大概处在向否定词语法化的起点, 还没 有连城客话和黄孝方言 “濑” 那种广泛的搭配能力。

6）卢著在嘉禾土话语料中用了 “没” 和 “不” 两种写法, 分别对应两大类用法, 但读音 却都是完全相同的 $\left[\mathrm{me}^{22}\right]$ 。承卢博士惠告, 原稿都作 “没”, “不” 是出版社编转的不当 改动, 应当统一作 “没”。

7）如苏州: [清] 《文武香球》“行豆才无得末, 那択讨饭吓一一行头都没有的话, 怎么 要饭呢? 引自石汝杰 1996:49。上海: 《方言圣人行实摘录 - 圣类思公撒格》, 1913 年: 想得出个苦工无得一样勿做到。

8) 北京话单独成句的否定词、形容词、副词也不是完全不荋要助句要索。在上海师大演 讲时, 有听讲者提醒笔者, 单独成句的这些词语需要语调上的加强和重读, 假如带上其 他成分, 则不必这么念。确实如此。如“你明天去吗? 一不。/不去。” “他长得怎么样? 帅。/ 很帅。”两种回答中, 单说 “不”、“帅”必须重读并带上较强的降调, 而 “不去”、 “很帅” 中的 “不”、“帅” 可以说得弱得多。因此可以说, 北京话可以单用的律手段帮 助这些词语成句, 而吴语还要有虚词等其他词汇句法手段才行。此外, 比起英语会话中 的 no 来, 北京口语中 “不” 单独成句要少得多, 张伯江先生提示我这一点。笔者对王 朔小说对话部分的初步考察也初步证明了这一点, 而且有些作品如《顶主》单独成句只 有连用的 “不不” (8 例), 没有 “不”, 在《过把當就死》中则是 “不不” (3 例)、“不 
不不”（1 例）和 “不”（2 例）并存。总体上这些包含大量口语对话的中篇小说中单独 成句的 “不” 的用例即使算上连用的“不不” 等也非常有限。

\section{〈照文献>}

曹志耘・秋谷裕幸・太田斋・赵日新 2000、「吴语处街方言研究」。日本好文出版「开篇」 单刊 No.12。

陈满华 1995. 【安仁方言」。北京语言学院出版社。

陈淑梅 2001.【郖东方言语法研究」。江苏教育出版社。

陈泽平 1998. 福州方言研究」。福建人民出版社。

本启群 2002.【吉首方言研究」。民族出版社。

本 荣主编 2002.【现代汉语方言大词典】。江苏教育出版社。温州分卷本作者游汝杰、杨 乾明; 宁波分卷本作者汤珍珠、陈忠敏、吴新贤。

㞭如龙 2001. 闽南方言的否定词和否定式」, “中国东南部方言比较研究” 第 8 次国际研 讨会, 上海。

力提甫. 托乎提主编 2002.【河尔泰语言学导论」。山西教育出版社。

林立芳 1997. 【梅县方言语法论稿」。中华工商联合出版社。

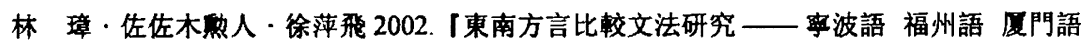
の分析」。日本好文出版「开篇」单刊No.13。

刘丹青 2001.「语法化中的更新、强化与加」, 「语言研究」第 2 期。

-2002.「上海方言否定词与否定式的文本统计分析」, 北京大学「语言学论丛」 26

辑, 商务印书馆。

刘俐李 1989.「回民乌鲁木齐语言志」。新壁大学出版社。

刘月华 1980.「可能补语用法的研究」，「中国语文」第 4 期。

卢小群 2002.【嘉禾土话研究」。中南大学出版社。

潘俉云 2002. 「汉语否定词考源一一兼论虚词考本字的基本方法」，「中国语文」第 4 期。

平田昌司主编 1998.【微州方言研究」。日本好文出版【开篇」单刊 No.9。

钱乃荣 1992.【当代吴语研究」。上海教育出版社。

钱曾怡主编 2001.【山东方言研究」。齐鲁书社。

桥本万太郎著, 余志鸿译 1985. 语言地理类型学」。北京大学出版社。日文原版 1978 年。

覃远雄 2003. 「汉语方言否定词的读音」, 「方言」第 2 期。

石汝杰 1996.【吴语读本】。日本好文出版「开篇】单刊 No.8。

太田辰夫 2003. 蒋绍雭、徐昌华译「中国语历史文法 (修订译本)」, 北京大学出版社。日

文原版 1958 年。

王森 2001. 「东干话的语序」, 「中国语文了第 3 期。

吴福样 2003. 「南方方言能性述补结构 “V 得 / 不 C” 带宾语的语序类型」,「方言」第 3 期。 
项梦冰 1997. 「连城客家话语法研究】。语文出版社。

詹伯慧主编 2002. 「广东方言概要」。雵南大学出版社。

张敏 2002. 「上古、中古汉语及现代南方方言里的 “存在 - 否定演化圈”」, 载

Proceedings of International Symposium on the Historical Aspect of the

Chinese Language: Commemorating the Centennial Birthday of the Late Professor Li Fang-Kuei, Vol II. pp. 571-616. Edit by Anne Yue. University of Washington, Seattle.

Dryer, Matthew 1988. Universals of negative position. In Hammond, Micheal, Edith Moravicsik \& Jessica Wirth (eds.) Studies in Syntactic Typology. Amsterdam: Benjamins.

Givón T. 1984. Syntax: A Functional-Typological Introduction. Vol. I. Amsterdam / Philadelphia: John Benjamins Publishing Company.

Kortmann, Bernd 1999. Typology and Dialectology. In Proceedings of the 16th International Congress of Linguists. CDRom, Amsterdam: Elsevier Science. 中文本刘海燕 译, 刘丹青校注, 《类型学与方言学》、《方言》2004 年第 2 期。

Palmer, F. R. 1974. The English Verb. London: Longman. 1st. Edition titled as $A$ Linguistic Study of the English Verb, 1965.

Trask, P. L. 1996/2000 Historical Linguistics. Edward Amold (Publishers) Ltd. 1996. 据外语教 学与研究出版社 2000 年中国大陆引进版。

\section{<附记>}

本文初稿是笔者在日本中国语学会第 54 回全国大会 “汉语中的否定范捧” 专题会的 大会报告。承蒙京都大学文学部田洼行则教授、日本中国语学会的邀请和京都大学的资 助, 我得以参加此会, 获得很多教益。另蒙沈力教授辛苦担任演讲时的日语同声翻译, 并 对初稿提供有益意见。本项研究也得到中国社科院重点课题 “汉语方言语法类型比较与方 言语法语料库” 的资助，博士生唐正大提供关中方言调查材料。会后，此文又在南开大学、 河北师范大学、中国社会科学院语言研究所和上海师范大学作过报告, 得到不少宝㭧意 见。此次修改吸收了各次讲演中听到的一些意见。谨向以上先生、机构及提出意见的同行 一并致谢。尚存的问题均由笔者本人负责。 